\title{
CO desorption from a catalytic surface: Elucidation of the role of steps by velocity- selected residence time measurements
}

Kai Golibrzuch ${ }^{1,2}$, Pranav R. Shirhatti ${ }^{1,2}$, Jan Geweke ${ }^{1,2}$, Jörn Werdecker ${ }^{1,2,4}$, Alexander Kandratsenka $^{1,2}$, Daniel J. Auerbach ${ }^{3}$, Alec M. Wodtke ${ }^{1,2,3}$ and Christof Bartels ${ }^{1,2}$

${ }^{1}$ Institute for Physical Chemistry, Georg-August University of Göttingen, Germany

${ }^{2}$ Max Planck Institute for Biophysical Chemistry, Göttingen, Germany

${ }^{3}$ Department of Chemistry and Biochemistry, University of California Santa Barbara

${ }^{4}$ Laboratoire Chimie Physique Moléculaire, École Polytechnique Fédérale de Lausanne (EPFL), CH-1015 Lausanne, Switzerland.

\section{Kinetic modeling of desorption rates}

For a first-order desorption kinetic assumption, the change of the number of adsorbed molecules, $N_{\text {ad }}$, per surface area is given by Eq. (S1):

$$
\frac{\mathrm{d} N_{\mathrm{ad}}(t)}{\mathrm{d} t}=\Phi_{i}(t)-k_{d}\left(T_{S}\right) N_{\mathrm{ad}}(t)
$$

where $\Phi_{i}(t)$ is the time dependent flux of molecules approaching the surface, modeled by the sum of two Gaussian functions (Eq. (S2)) obtained from a measurement at $T_{S}=973 \mathrm{~K}$ :

$$
\Phi_{i}(t)=A_{1} \mathrm{e}^{-\left(t-t_{1}\right)^{2} / w_{1}^{2}}+A_{2} \mathrm{e}^{-\left(t-t_{2}\right)^{2} / w_{2}^{2}}
$$

The number of molecules leaving the surface in a time interval $t \ldots t+\mathrm{d} t$ is given by the number of adsorbed molecules, $N_{\text {ad }}(t)$, at time $t$ multiplied with the rate constant, $k_{d}$, for desorption. We obtain the analytical expression (Eq. (S3)) for $N_{\mathrm{ad}}(t)$ by integration of Eq. (S1) for $N_{\mathrm{ad}}(0)=0$.

$$
\begin{aligned}
N_{\mathrm{ad}}(t)=\mathrm{e}^{-k_{d} t} \sqrt{\frac{\pi}{2}} & \left(A_{1} \mathrm{e}^{k_{d} t_{1}+\frac{k_{d}^{2} w_{1}^{2}}{2}} w_{1} \operatorname{Erf}\left(\frac{t_{1}+k_{d} w_{1}^{2}}{\sqrt{2} w_{1}}\right)\right. \\
& -A_{1} \mathrm{e}^{k_{d} t_{1}+\frac{k_{d}^{2} w_{1}^{2}}{2}} w_{1} \operatorname{Erf}\left(\frac{-t+t_{1}+k_{d} w_{1}^{2}}{\sqrt{2} w_{1}}\right) \\
& +A_{2} \mathrm{e}^{k_{d} t_{2}+\frac{k_{d}^{2} w_{2}^{2}}{2}} w_{2} \operatorname{Erf}\left(\frac{t_{2}+k_{d} w_{2}^{2}}{\sqrt{2} w_{2}}\right) \\
& \left.-A_{2} \mathrm{e}^{k_{d} t_{2}+\frac{k_{d}^{2} w_{2}^{2}}{2}} w_{2} \operatorname{Erf}\left(\frac{-t+t_{2}+k_{d} w_{2}^{2}}{\sqrt{2} w_{2}}\right)\right)
\end{aligned}
$$


Since the sticking probability is not unity, we add a direct scattering contribution with zero residence time to the flux of molecules leaving the surface (Eq. (S4)). The two additional factors $B_{\mathrm{DS}}$ and $B_{\mathrm{TD}}$ are used to scale the model to the signal size observed in the experiment.

$$
\Phi_{d}\left(t, T_{S}\right)=\underbrace{B_{\mathrm{DS}} \Phi_{i}(t)}_{\text {direct scattering }}+\underbrace{B_{\mathrm{TD}} k_{d}\left(T_{S}\right) N_{\mathrm{ad}}(t)}_{\text {desorption }}
$$

For the observation of bi-exponential desorption kinetics, Eq. (S4) changes to Eq. (S5):

$$
\Phi_{d}\left(t, T_{S}\right)=\underbrace{B_{\mathrm{DS}} \Phi_{i}(t)}_{\text {direct scattering }}+\underbrace{B_{\mathrm{TD}}^{\text {fast }} k_{d}^{\text {fast }}\left(T_{S}\right) N_{\mathrm{ad}}^{(1)}(t)}_{\text {fast desorption }}+\underbrace{B_{\mathrm{TD}}^{\text {slow }} k_{d}^{\text {slow }}\left(T_{S}\right) N_{\mathrm{ad}}^{(2)}(t)}_{\text {slow desorption }}
$$

The model now contains two different rate constants, $k_{d}^{\text {fast }}\left(T_{S}\right)$ and $k_{d}^{\text {slow }}\left(T_{S}\right)$, as well as two different kinds of adsorbates, $N_{\mathrm{ad}}^{(1)}(t)$ and $N_{\mathrm{ad}}^{(2)}(t)$. Their time dependent population is given by Eq. (S6) and Eq. (S7), respectively:

$$
\begin{aligned}
& N_{\text {ad }}^{(1)}(t) \\
& =\mathrm{e}^{-k_{d}^{\mathrm{fast}} t} \sqrt{\frac{\pi}{2}}\left(A_{1} e^{k_{d}^{\mathrm{fast}}} t_{1}+\frac{\left(k_{d}^{\mathrm{fast}}\right)^{2} w_{1}^{2}}{2} w_{1} \operatorname{Erf}\left(\frac{t_{1}+k_{d}^{\mathrm{fast}} w_{1}^{2}}{\sqrt{2} w_{1}}\right)\right. \\
& -A_{1} \mathrm{e}^{k_{d}^{\mathrm{fast}}} t_{1}+\frac{\left(k_{d}^{\mathrm{fast}}\right)^{2} w_{1}^{2}}{2} w_{1} \operatorname{Erf}\left(\frac{-t+t_{1}+k_{d}^{\mathrm{fast}} w_{1}^{2}}{\sqrt{2} w_{1}}\right) \\
& +A_{2} \mathrm{e}^{k_{d}^{\mathrm{fast}}} t_{2}+\frac{\left(k_{d}^{\mathrm{fast}}\right)^{2} w_{2}^{2}}{2} w_{2} \operatorname{Erf}\left(\frac{t_{2}+k_{d}^{\mathrm{fast}} w_{2}^{2}}{\sqrt{2} w_{2}}\right) \\
& \left.-A_{2} \mathrm{e}^{k_{d}^{\mathrm{fast}}} t_{2}+\frac{\left(k_{d}^{\mathrm{fast}}\right)^{2} w_{2}^{2}}{2} w_{2} \operatorname{Erf}\left(\frac{-t+t_{2}+k_{d}^{\mathrm{fast}} w_{2}^{2}}{\sqrt{2} w_{2}}\right)\right) \\
& N_{\mathrm{ad}}^{(2)}(t) \\
& =\mathrm{e}^{-k_{d}^{\text {slow }} t} \sqrt{\frac{\pi}{2}}\left(A_{1} e^{k_{d}^{\text {slow }}} t_{1}+\frac{\left(k_{d}^{\text {slow }}\right)^{2} w_{1}^{2}}{2} w_{1} \operatorname{Erf}\left(\frac{t_{1}+k_{d}^{\text {slow }} w_{1}^{2}}{\sqrt{2} w_{1}}\right)\right. \\
& -A_{1} \mathrm{e}^{k_{d}^{\text {slow }}} t_{1}+\frac{\left(k_{d}^{\text {slow }}\right)^{2} w_{1}^{2}}{2} w_{1} \operatorname{Erf}\left(\frac{-t+t_{1}+k_{d}^{\text {slow }} w_{1}^{2}}{\sqrt{2} w_{1}}\right) \\
& +A_{2} \mathrm{e}^{k_{d}^{\text {slow }}} t_{2}+\frac{\left(k_{d}^{\text {slow }}\right)^{2} w_{2}^{2}}{2} w_{2} \operatorname{Erf}\left(\frac{t_{2}+k_{d}^{\text {slow }} w_{2}^{2}}{\sqrt{2} w_{2}}\right) \\
& \left.-A_{2} \mathrm{e}^{k_{d}^{\text {slow }}} t_{2}+\frac{\left(k_{d}^{\text {slow }}\right)^{2} w_{2}^{2}}{2} w_{2} \operatorname{Erf}\left(\frac{-t+t_{2}+k_{d}^{\text {slow }} w_{2}^{2}}{\sqrt{2} w_{2}}\right)\right)
\end{aligned}
$$

